\title{
Evaluación del potencial del desarrollo en ciencia y tecnología en México 2000-2015
}

Assessing the potential of science and

technology development in México: 2000-2015

\author{
Rafael Buendía García * \\ Jorge Pablo Rivas Díaz ** \\ Ireiri Alonso León ${ }^{* * *}$
}

\section{Resumen}

El presente artículo permite reconocer el potencial de desarrollo de la Ciencia y la Tecnología (CyT) en México, entre 2000 y 2015. A partir de la esquematización de un Índice de Potencial de Desarrollo en Ciencia y Tecnología que considera: 1) el sistema educativo en la formación de recursos humanos, 2) la realización de un sistema interno de producción científica y tecnológica, 3) el nivel de inversión en desarrollo científico y tecnológico, 4) la dimensión y dinamismo en la producción científica y tecnológica y 5) el grado de capitalización de los factores de la producción en ingresos y crecimiento económico; como determinantes para analizar los avances y retrocesos en materia de ciencia y tecnología. A partir del Índice, se observa una desarticulación entre los recursos humanos y la capitalización, que a nivel nacional lleva a tener grandes
* Profesor adscrito a la División de Sistema de Universidad Abierta y Educación a Distancia de la Facultad de Economía, UNAM

**Profesor adscrito a la División de Estudios Profesionales de la Facultad de Economía, UNAM

****Estudiante de la Licenciatura en Economía de la Facultad de Economía, UNAM

Palabras clave Cambio tecnológico, Gestión e innovación tecnológica, Política gubernamental

Key words Tecnological Change, Management of Tecnological Innovation, Government Policy JEL $03,032,038$ 
desigualdades, mermando el potencial de desarrollo. Además, se consideran seis limitantes al progreso del potencial en CyT: 1) desequilibrios en la distribución espacial, 2) desarticulación de las etapas en la formación educativa, 3) recursos humanos especializados, escasos y desequilibrados, 4) producción baja y desarticulada de las prioridades nacionales; 5) baja inversión y gasto público ineficiente y, 6) baja capitalización y dependencia tecnológica. Estos límites generan que aumente la concentración y desigualdad en el potencial del desarrollo de la CyT, teniendo un impacto negativo en la economía nacional, y posicionándonos en un nivel bajo a nivel internacional.

\section{Abstract}

The present article allows to recognize the potential of development of Science and Technology (S\&T) in Mexico between 2000 and 2015. The schematization of a Development Potential Index in Science and Technology considers: 1) educational systems in the formation of human resources, 2) the realization of an internal system of scientific and technological production, 3) a level of investment in scientific and technological development, 4) the dimension and dynamism in the scientific and technological production and 5) the degree of capitalization of production factors in income and economic growth; as determinants to analyze the advances and setbacks in science and technology. From the Index, there is a disarticulation between human resources and capitalization, which at national level leads to large inequalities, reducing the potential for development. In addition, six constraints are considered to the potential progress in $\mathbf{S}$ \& T: 1) imbalances in spatial distribution, 2) disarticulation of the stages in the educational formation, 3) special, scarce and unbalanced human resources, 4) low and disjointed production of national networks, 5) low investment and inefficient public spending, and 6) low capitalization and technological dependence. These limits generate concentration and inequality in the potential of S\&T development, having a negative impact on the national economy, and positions at an internationally low level.

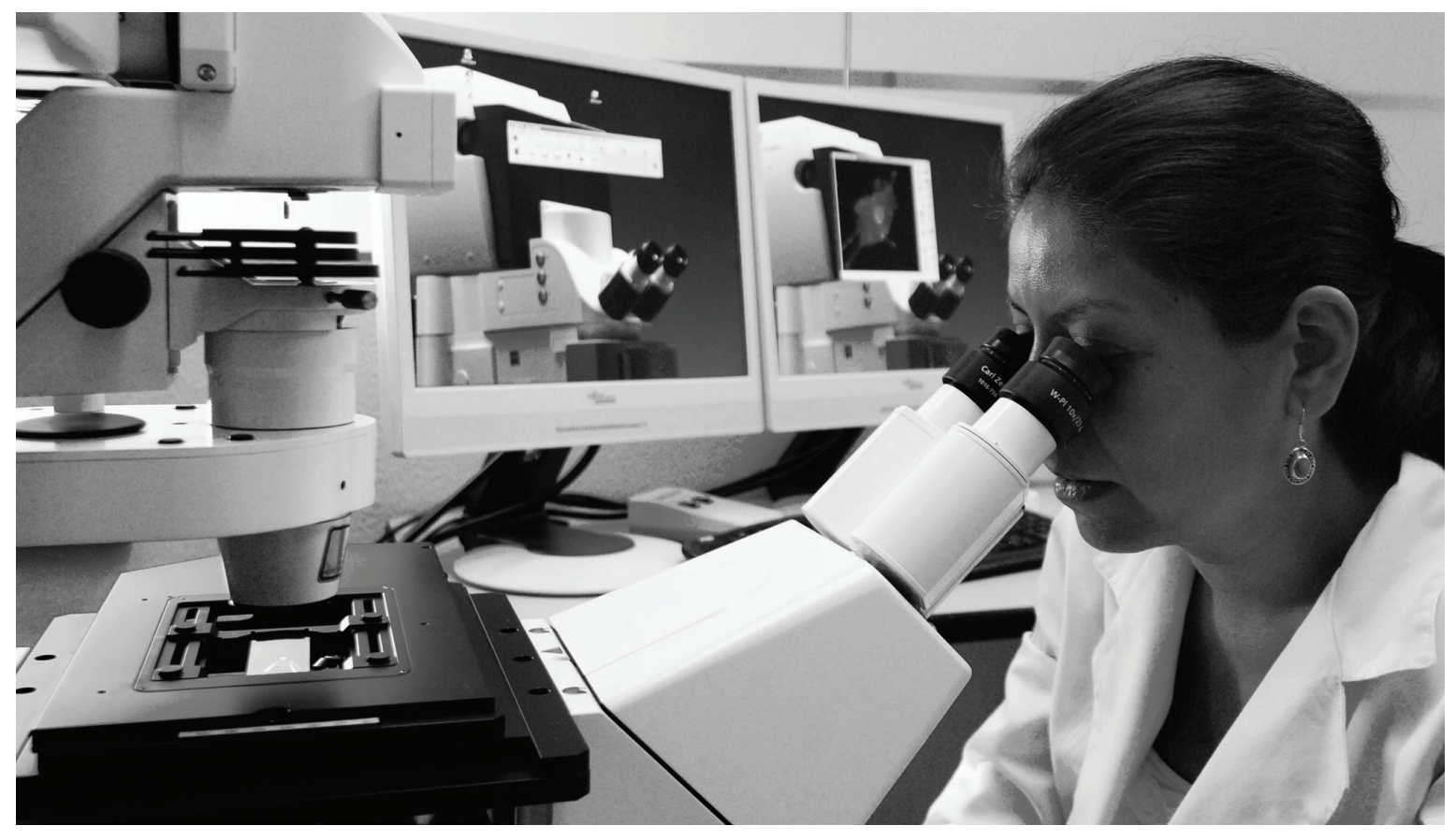


Rafael Buendía García | Jorge Pablo Rivas díaz | Ireiri Alonso León|

Evaluación del potencial del desarrollo en ciencia

y tecnología en México 2000-2015

\section{Introducción}

A lo largo de la historia, el desarrollo del conocimiento y su aplicación a la solución de los problemas específicos de la sociedad, tiene como consecuencia un efecto en cadena que lleva gradualmente al incremento de la producción, el consumo y la generación de riqueza por parte de los agentes económicos. Dichos conocimientos se traducen en la implementación de ciencia y tecnología.

El objetivo de ésta investigación es analizar el potencial de desarrollo de la Ciencia y la Tecnología en México en el periodo 2000 y 2015, como pilares para entender el grado de crecimiento económico.

El primer apartado de este trabajo consiste en una primera aproximación de la Ciencia y la Tecnología como determinantes del progreso económico de los estados nacionales, así como los factores que condicionan su desarrollo.

En el segundo apartado se esquematiza el Índice de Potencial de Desarrollo en Ciencia y Tecnología, el cual a su vez engloba cinco determinantes principales: cobertura educativa, recursos humanos especializados, inversión en Investigación y Desarrollo, producción científica y capitalización de los factores de la producción; los cuales permiten evaluar el progreso potencial entre los distintos estados de la República Mexicana.

Finalmente, a partir del diagnóstico del Índice de Potencial de Desarrollo en Ciencia y Tecnología, se presentan seis limitaciones que condicionan el progreso a nivel estatal y nacional.

\section{El papel de la Ciencia y la Tecnología en el progreso económico}

La Ciencia y la Tecnología (CyT) son dos conceptos que han tomado gran relevancia en el análisis de la economía actual. Por un lado, el desarrollo científico se refiere a la generación de conocimientos nuevos provenientes de la observación, experimentación y comprobación. Por otro lado, la tecnología implica la resolución de problemas prácticos que afectan a la sociedad y sus agentes económicos a través de la aplicación de nuevos conocimientos generados desde la ciencia (Velho, 2011).

El análisis de las causas y efectos del progreso de la CyT está presente en el desarrollo del pensamiento económico, en su búsqueda por explicar el progreso de la sociedad en su conjunto y el funcionamiento del sistema productivo.

La división social del trabajo, acompañada del desarrollo de la maquinaria industrial, han sido planteados como factores fundamentales del crecimiento de la productividad y la generación de la riqueza (Smith, 1997). Se argumenta que la especialización productiva, acompañada del progreso tecnológico resultan mecanismos necesarios para incrementar y canalizar el comercio internacional hacia los productores dotados de ventajas competitivas (Ricardo, 2003).

Así mismo, se ha desarrollado el pensamiento de que la relación proporcional de la tecnología dentro los procesos productivos en los distintos sectores económicos, genera un alto nivel de la productividad del trabajo y el crecimiento económico, como factores explicativos del consumo y la inversión (Marx, 1999).

Actualmente, se presentan dos grandes ideales acerca del papel de la CyT en el análisis económico.

Primero, un análisis desde el enfoque macroeconómico, donde se canaliza el estudio en la argumentación de los mecanismos del incremento de la productividad del trabajo y su relación con el crecimiento económico; el papel de la tecnología en la sustitución de los factores de la producción y la reducción 
de los costos de la producción; también en las formas mediante las cuales se transfiere el efecto de la innovación y la tecnología entre los distintos sectores económicos (Kim, Loayza, \& Meza-Cuadra, 2016).

Segundo, un análisis desde la perspectiva microeconómica de la organización industrial, que desarrolla conceptos sobre las causas de la investigación y desarrollo anclado a las necesidades de la rentabilidad de las empresas; aportaciones relacionadas a la sistematización de los mecanismos específicos de la difusión de la innovación a través de las empresas y la generación de sinergias industriales alrededor de las empresas más innovadoras; además del papel de los factores condicionantes para el desarrollo acelerado de la $\mathrm{CyT}$, como son las institucionales locales y regionales que incentivan la actividad innovadora, la existencia de infraestructura física para el desarrollo científico y tecnológico; y el papel del capital humano como eje principal en el proceso innovador (ocDE, 2012).

La concepción acerca de que el progreso científico y tecnológico, es decir del desarrollo continuo de los medios de producción como base de la dinámica económica, del crecimiento de la producción y su expansión internacional, resulta una idea cada vez más común y poderosa, impulsada desde los principales organismos internacionales orientados al desarrollo económico (Reamer, 2014).

\section{Índice de Potencial de}

\section{Desarrollo en Ciencia y Tecnología}

Para realizar un diagnóstico sobre los avances y retrocesos en materia de CyT se recurren a herramientas cuantitativas, por ello se parte de cinco determinantes que el potencial de desarrollo de la CyT: 1) el sistema educativo en la formación de recursos humanos, 2) la realización de un sistema interno de producción científica y tecnológica, 3) el nivel de inversión en desarrollo científico y tecnológico, 4) la dimensión y dinamismo en la producción científica y tecnológica y 5) el grado de capitalización de los factores de la producción en ingresos y crecimiento económico.

De tal forma que el potencial de desarrollo para la CyT se esquematiza en un sólo índice global, de la siguiente manera:

Sea el IPDCyT, el Índice de Potencial de Desarrollo en Ciencia y Tecnología para los estados de la República, tal que:

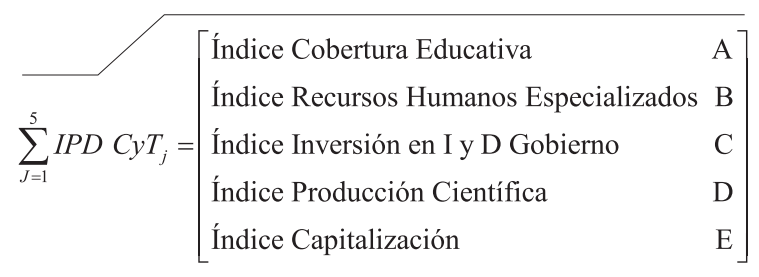

Donde (A,B,C,D y E) simbolizan los cinco determinantes, definidos como componentes primordiales del IPDCyT, ponderado de manera igual entre sus cinco dimensiones; cada uno determinado a través del complemento de 14 indicadores específicos ajustados por el método de estandarización lineal simple contra parámetros de progreso ideal a nivel internacional.

Por consiguiente, se puede formular el planteamiento general del IPDCyT para evaluar el potencial de desarrollo mediante la siguiente ecuación:

$$
I P D C y T_{j}=\sum_{J=1}^{5} F I M_{j} \frac{n j}{100} * \sum_{j=1}^{n j} V A_{j i} F I_{j i}
$$


Rafael Buendía García | Jorge Pablo Rivas díaz | Ireiri Alonso león|

Evaluación del potencial del desarrollo en ciencia

y tecnología en México 2000-2015

Donde:

$\operatorname{FIM}_{j} \frac{n j}{100}=$ Factor de peso del j-ésimo Índice de Dimensión

$V A_{j i}=$ Valor del i-ésimo Índice ponderado perteneciente al j-ésimo Índice de Dimensión

$F I_{j i}=$ Factor de peso del i-ésimo Índice ponderado perteneciente al j-ésimo Índice de Dimensión

$n j$ = número de índices ponderados del j-ésimo Índice mayor

Donde además cada Índice ponderado se encuentra definido en indicadores específicos, estandarizados linealmente, tal que:

$$
V A_{j i}=\sum_{k=1}^{n_{i j}} V S I_{j i k} F S I_{j i k}
$$

Donde:

$V S I_{j i k}=$ Valor del k-ésimo indicador específico del i-ésimo Índice ponderado
$F S I_{j i k}=$ Factor de peso del k-ésimo indicador específico del i-ésimo índice ponderado

nij = número de indicadores específicos del iésimo Índice ponderado

La evaluación del IPDCyT presentada en el cuadro 1 , muestra que a nivel nacional existe un progreso agregado del $21 \%$ entre el año 2000 y 2015. La magnitud de este avance ha generado un escalamiento al trasladar a México de un nivel de potencial muy bajo, equivalente a 0.226 a uno bajo equivalente a 0.286 , para el mismo periodo.

El análisis de los limitantes al progreso de la CyT, así como la evaluación del bajo nivel de su desarrollo y la baja tendencia hacia su progreso, se encuentra en el análisis de sus determinantes y sus relaciones funcionales. Cada limitante está al interior de una de las cinco categorías de sus determinantes; donde las tendencias y las dinámicas de sus elementos resultan desarticuladas o incluso contradictorias entre sí, lo cual es producto de la interacción específica de sus

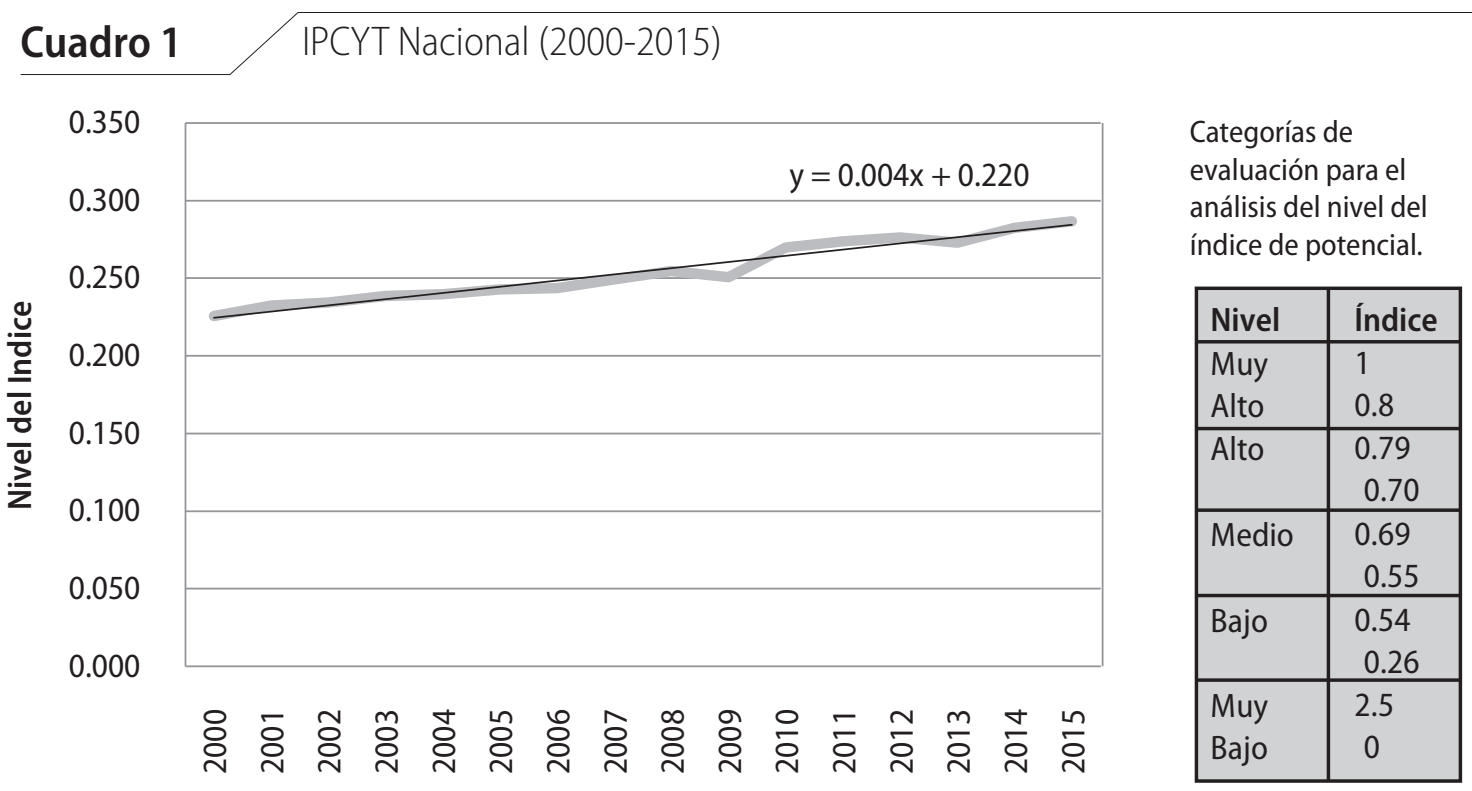

Fuente: elaboración propia basada en el modelo de índice compuesto "Índice de Potencial de Desarrollo en Ciencia y Tecnología", con fuentes Nacionales e Internacionales de indicadores en C y T expuestas durante la investigación. 


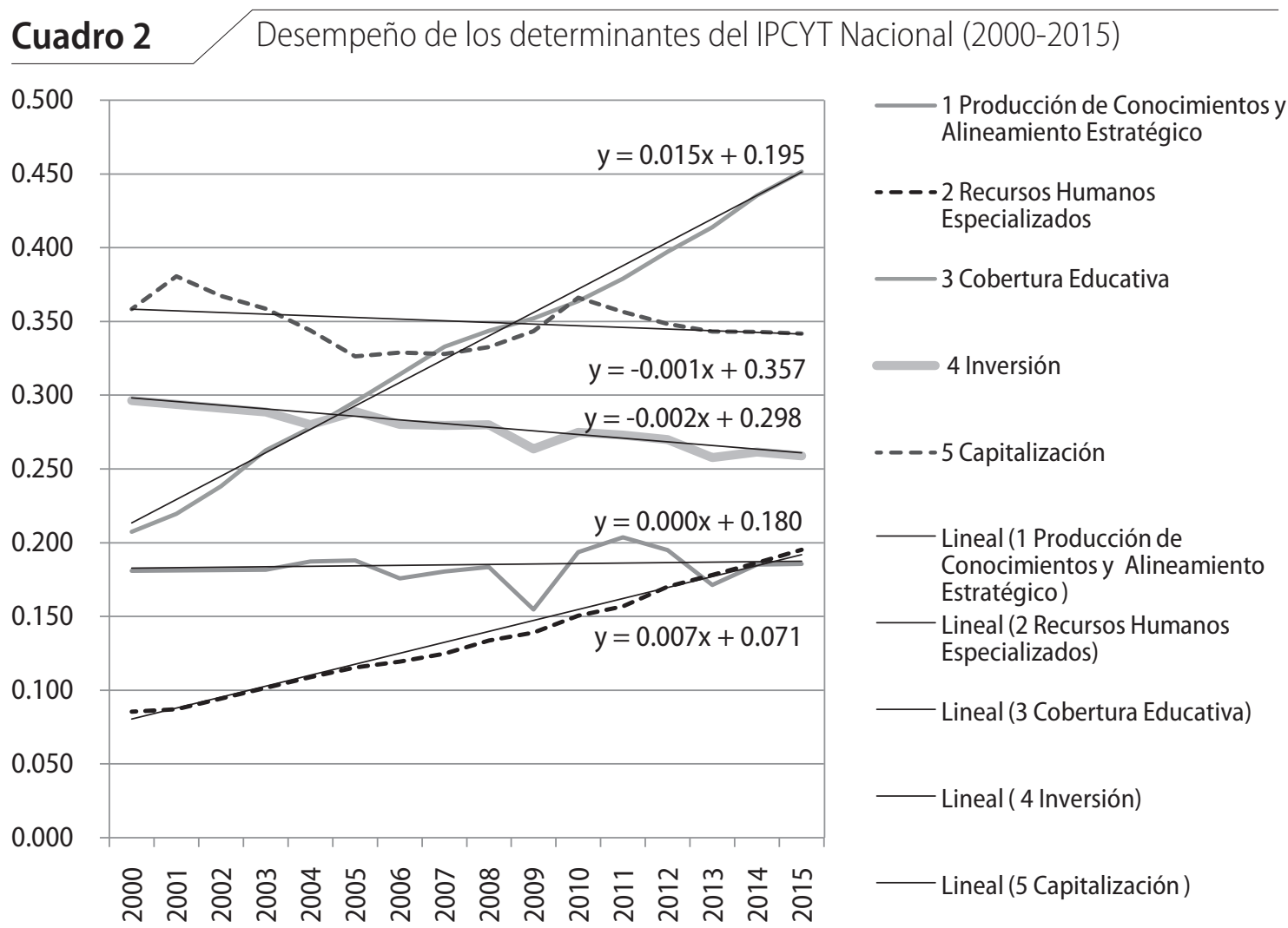

Nota: Donde $Y$ es el nivel de potencial de desarrollo en ciencia y tecnología observado para los diferentes años, en los distintos rubros.

La ecuación asociada a cada serie histórica corresponde a una proyección lineal de la tendencia de la misma serie, véase por ejemplo el caso de la línea 4 (inversión) cuyo resultado es y=-0.0025x+0.2985, lo que significa que se observa una tendencia decreciente en la variable (inversión) conforme progresa el tiempo, que resulta para el año 2015 que el nivel máximo alcanzado a nivel nacional corresponde a un tercio del potencial máximo esperado a nivel internacional.

Fuente: elaboración propia basada en el modelo de índice compuesto “Índice de Potencial de Desarrollo en Ciencia y Tecnología", con fuentes Nacionales e Internacionales de indicadores en C y T expuestas durante la investigación.

elementos. En el cuadro 2 se muestran las series de índices calculados a nivel nacional por cada una de las dimensiones de determinantes del potencial en CyT, lo que permite revelar una proyección lineal del comportamiento futuro de los indicadores y medir su desempeño.

Se observa que entre 2000 y 2015 , la más alta variación absoluta observada fue en la dimensión de la producción de recursos humanos altamente especializados, la cual varió $128 \%$, incrementando la magnitud del potencial de desarrollo desde un 0.086 en el año 2000, a un 0.195 en 2015. Sin embargo, dicha variación no supera el nivel de desarrollo "muy bajo" si se compara a nivel internacional.

Por otro lado, el segundo lugar en el desempeño corresponde al progreso en la capacidad de formación de recursos humanos, a través del progreso de la cobertura educativa, con un avance de $118 \%$ durante el mismo lapso, escalando de un nivel de desarrollo muy bajo (0.20), a un nivel bajo (0.45).

Así mismo, la producción científica y tecnológica progresó, pero no logró llegar a un mejor nivel, teniendo una variación absoluta 
Rafael Buendía García | Jorge Pablo Rivas díaz | Ireiri Alonso León|

Evaluación del potencial del desarrollo en ciencia

y tecnología en México 2000-2015

de 3\%, pasando de un nivel acumulado de un 0.181 en 2000 a un 0.185 en 2015 .

Por el contrario, la inversión y el grado de capitalización de la CyT ha tenido descensos en sus variaciones totales de $-13 \%(2000)$ y $-4 \%$ (2015). A pesar de ello, se colocan dentro del rango del nivel de potencial de desarrollo "bajo", un nivel superior al de en la producción científica y la formación de recursos humanos especializados.

En consecuencia, el progreso en el potencial agregado a nivel estatal denota un desempeńo diferente entre los estados de la Repúbli- ca como lo muestra el cuadro 3 . Se observa un progreso en todos, pero un gran diferencial en la Ciudad de México, lo cual representa desequilibrios.

El mapa 1, muestra las diferencias del potencial de desarrollo de CyT, donde estados como la Ciudad de México (46\%), Querétaro (45\%), Baja California y Quintana Roo (38\%) y Zacatecas (37\%), son los más avanzados, mientras que Guerrero (8\%), Nayarit (9\%), Oaxaca (10\%), y Campeche (13\%) tienen progresos inferiores a $1 \%$ anual sobre el índice agregado.

\section{Cuadro 3 Progreso del IPCYT por estado de la República. Comparativo 2000 y 2015}

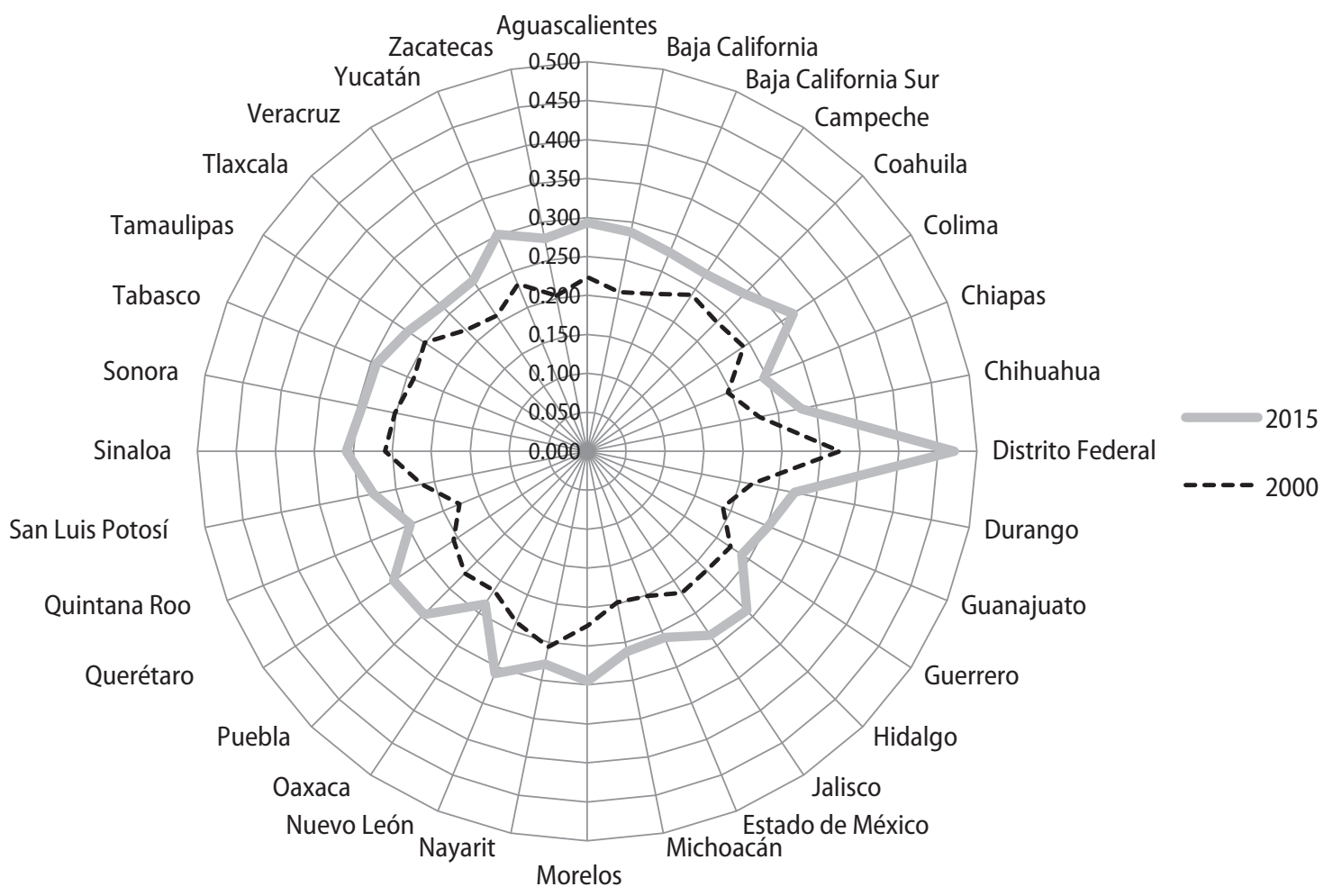

Fuente: elaboración propia basada en el modelo de índice compuesto "Índice de Potencial de Desarrollo en Ciencia y Tecnología", con fuentes Nacionales e Internacionales de indicadores en CyT expuestas durante la investigación. 


\section{Mapa 1 Progreso acumulado del IPDCyT (2000-2015) por entidad federativa}

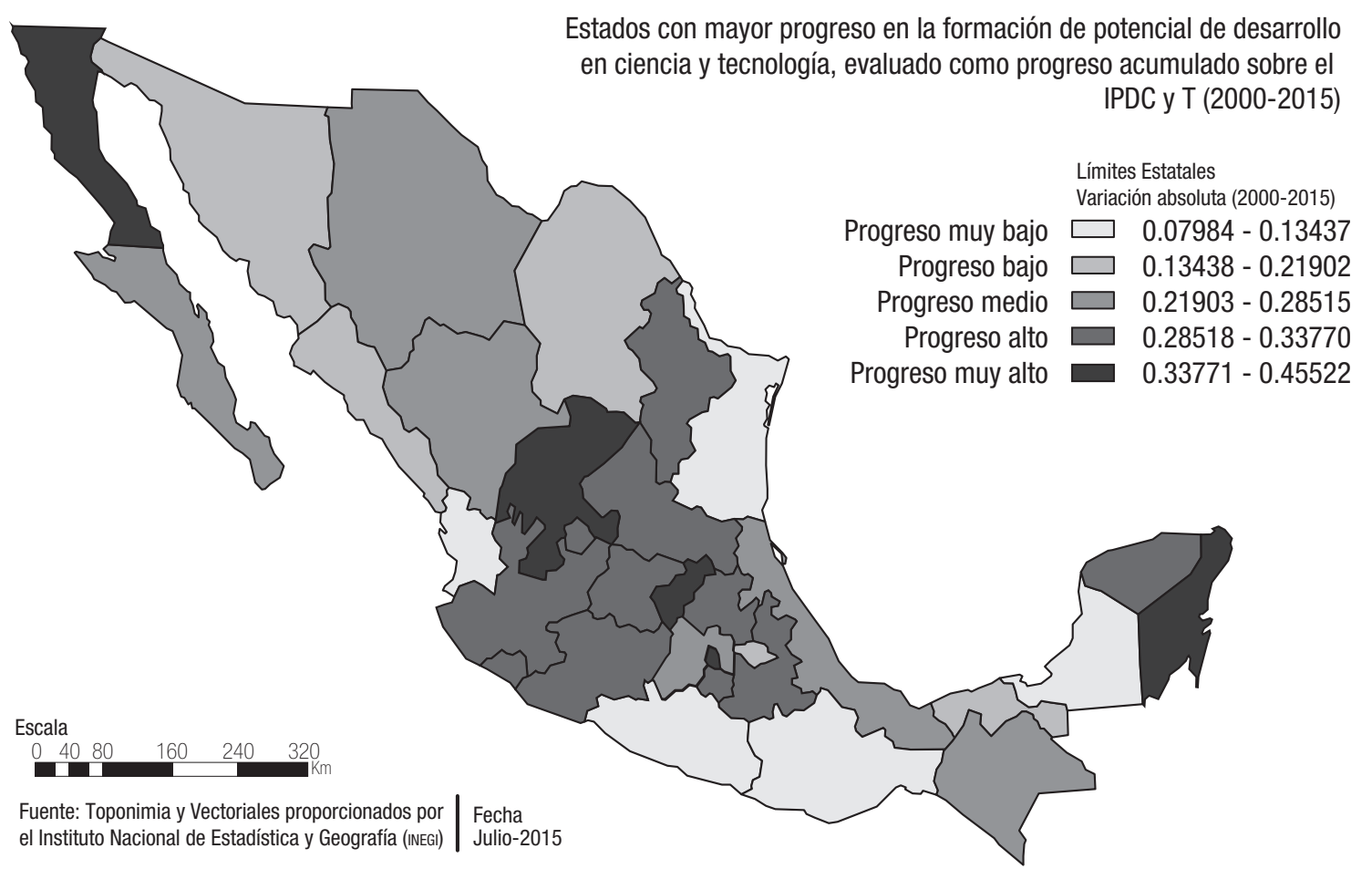

Por otra parte, el nivel de potencial por estado en 2015 se promediaba en 0.286 , lo que corresponde a una posición bajo respecto a estándares internacionales. De la misma manera la Ciudad de México sobresale, alcanzando un 0.471 que le permite aproximarse al nivel medio internacional. Este desempeño sobresale por mucho dentro de la tendencia nacional, donde le siguen los estados de Colima (0.318), Sinaloa (0.310), Nuevo León (0.309), Yucatán (0.301) y Querétaro (0.299).

En el mapa 2 se observa un desequilibrio estatal en el nivel de potencial de desarrollo en CyT de manera agregada, donde la Ciudad de México duplica el potencial de entidades como Oaxaca y Guerrero, con los peores niveles a nivel nacional.

\section{Limitantes del progreso del potencial de desarrollo en Ciencia y Tecnología}

A partir del análisis anterior, se puede argumentar que existen 6 limitantes al progreso del potencial de desarrollo en CyT a nivel estatal y nacional:

\section{Desequilibrios en la distribución espacial}

El potencial de desarrollo analizado por factores de la producción cuenta con una distribución espacial desequilibrada a nivel nacional, debido a que en la Ciudad de México hay una gran concentración de recursos humanos especializados, y las principales instituciones educativas y centros de investigación a nivel 


\section{Mapa 2 Niveles de potencial de desarrollo: IPDCyT 2015 por entidad federativa}

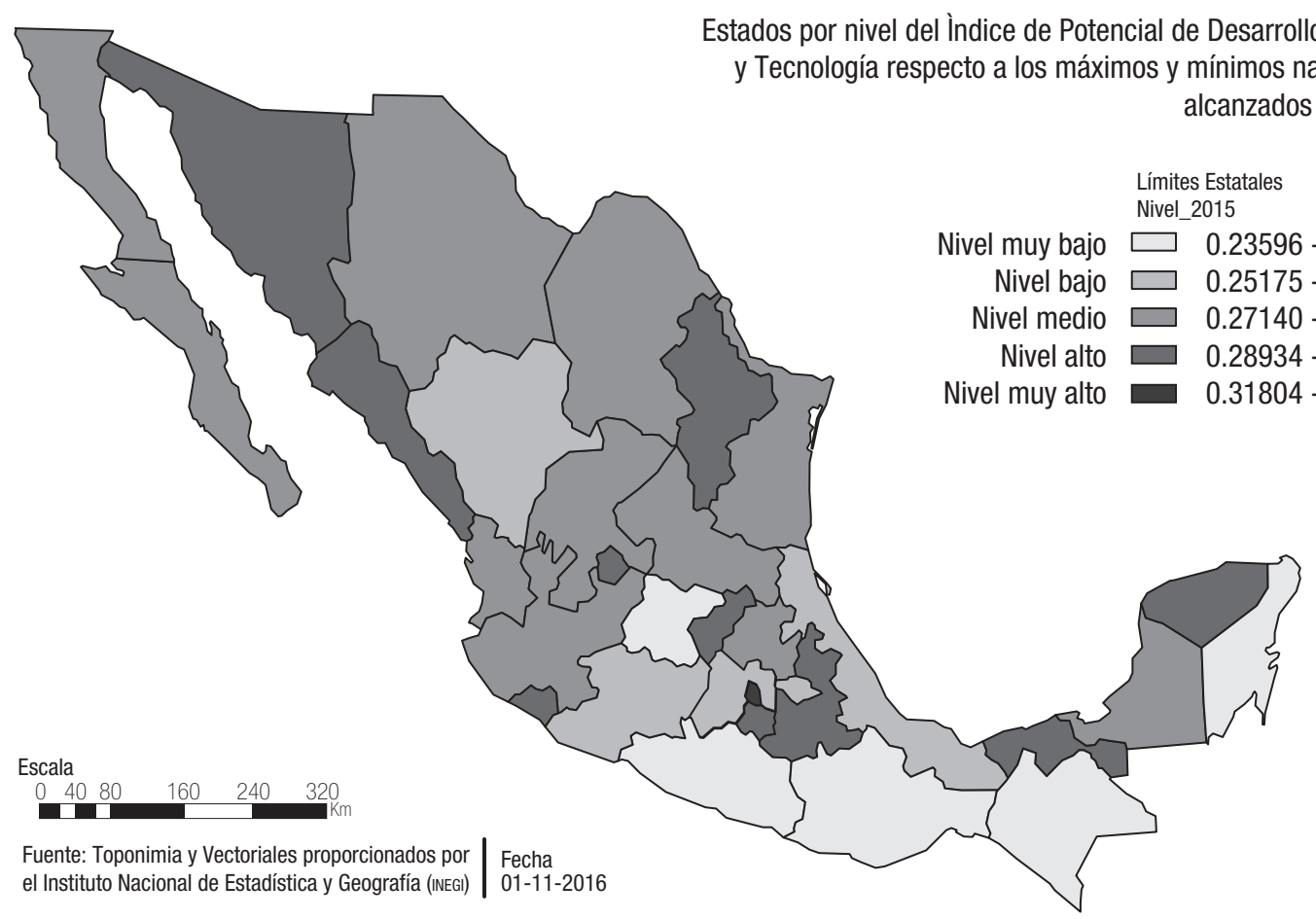

nacional. Así mismo, el capital invertido en la consolidación de la producción científica y tecnológica, así como su administración, tiene un alto grado de concentración espacial en la Ciudad de México.

Por otra parte, los recursos naturales explotables que pueden ser transformados para fines de la producción económica, se encuentran muy concentrados en lugares distintos al de las concentraciones del factor trabajo.

El mapa 3 muestra la desarticulación de los factores de la producción por su localización entre los distintos estados de la República, donde los estados con mayores riquezas naturales como Guerrero, Oaxaca y Chiapas, carecen de recursos financieros y humanos para desencadenar procesos productivos y crecimiento económico. Por el contrario, algunos estados como Nuevo León y la Ciudad de Mé- xico cuentan con mayores recursos humanos y financiero pero presentan gran insuficiencia de factores naturales de la producción.

\section{Desarticulación de las etapas en la formación educativa}

La evaluación de la calidad de la educación resulta un tema complejo, puesto que sólo se puede abordar desde un enfoque cuantitativo. Para 2000 a 2015, el nivel básico se cuenta con un promedio de cobertura nacional de $92 \%$, en el nivel medio superior desciende hasta un 58\% y el nivel superior se presenta una cobertura promedio de $24 \%$. Esto significa una baja de $30 \%$ promedio por nivel de progreso educativo, lo que implica un límite a la formación futura de recursos humanos especializados y la posibilidad de formar investigadores y científicos. 

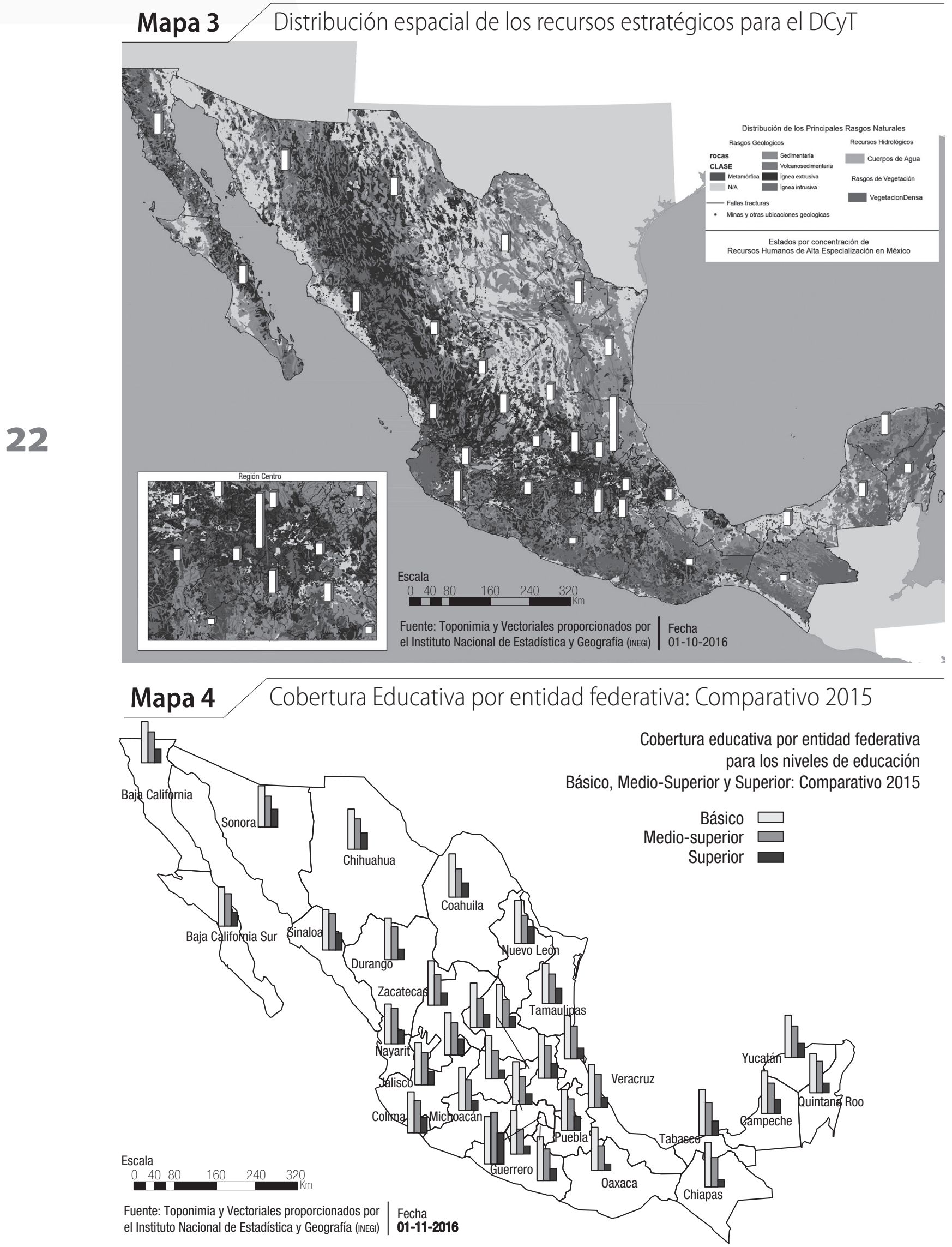
En el mapa 4 resalta la alta concentración geográfica y por ingresos en el acceso a la educación en los niveles medio superior y superior, lo que nuevamente disminuye el potencial de investigación de la sociedad mexicana, al reducir y concentrar la masa y características socioeconómicas de los estudiantes con mayores niveles educativos.

\section{Recursos humanos especializados, escasos y desequilibrados}

Entre 2000 y 2015, la educación superior y posgrados no superó $30 \%$ a nivel nacional, lo que significa que la cobertura en educación tecnológica y científica es baja y altamente concentrada, por tanto, representa otro límite a la formación interna de nuevos investigado- res. Además, disminuye la atracción de inversiones y la mejora del mercado laboral debido a la escasez de recursos humanos calificados. El determinante que explica ésta tendencia es la capacidad financiera de las familias para sostener la carrera educativa de los jóvenes, porque desincentiva la formación en las áreas científica y tecnológica. Esto se intenta superar mediante becas educativas, liberando estudiantes altamente productivos hacia la especialización de dichos conocimientos.

En la economía nacional, la escasez de formación de investigadores como recurso humano especializado, genera un atraso en dichas áreas de conocimiento. El mapa 5 muestra una distribución nacional desigual entre los distintos estados de la República, lo que limita el potencial de desarrollo.

\section{Mapa 5 Recursos Humanos Especializados por entidad federativa, 2015}

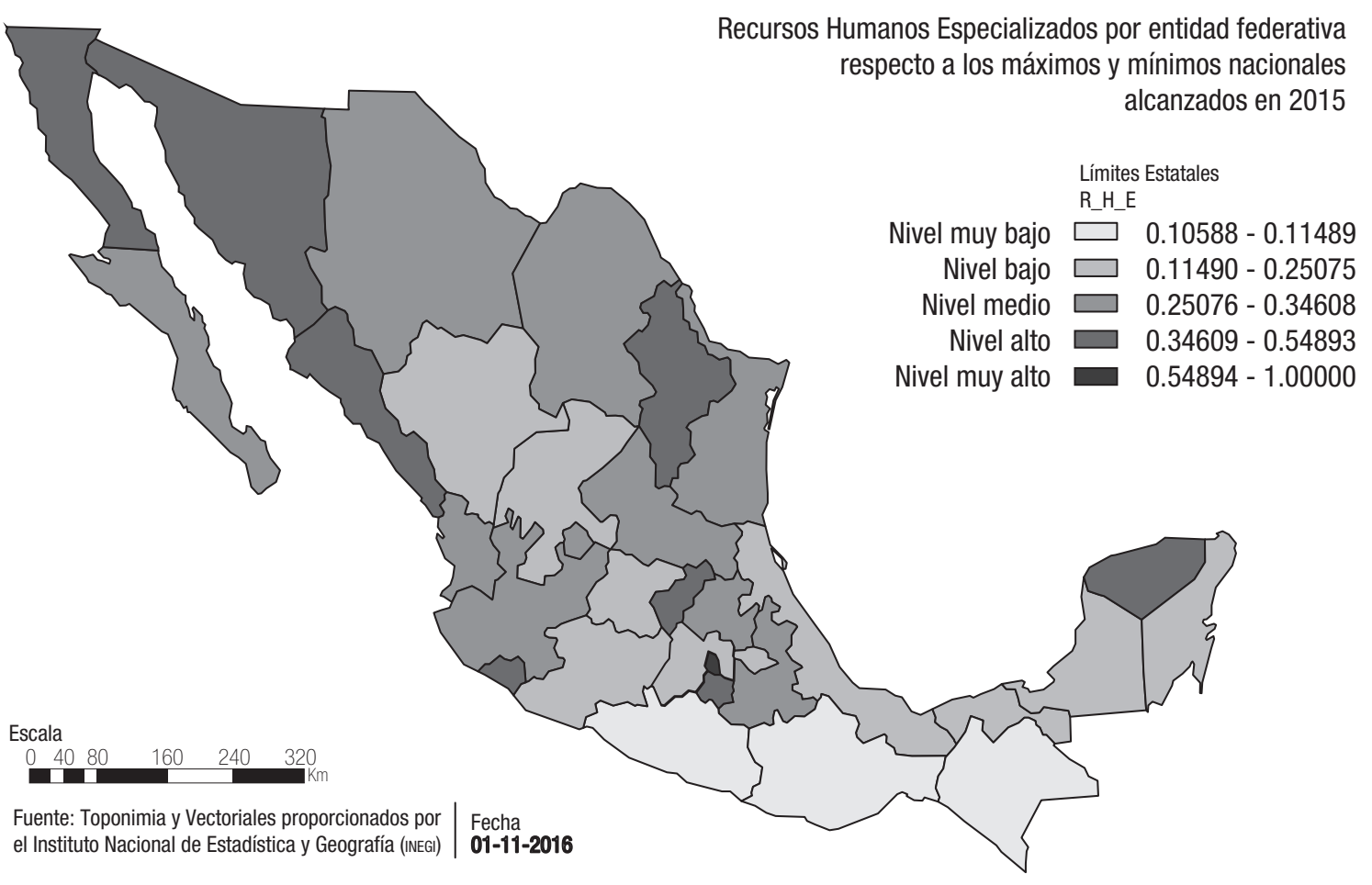




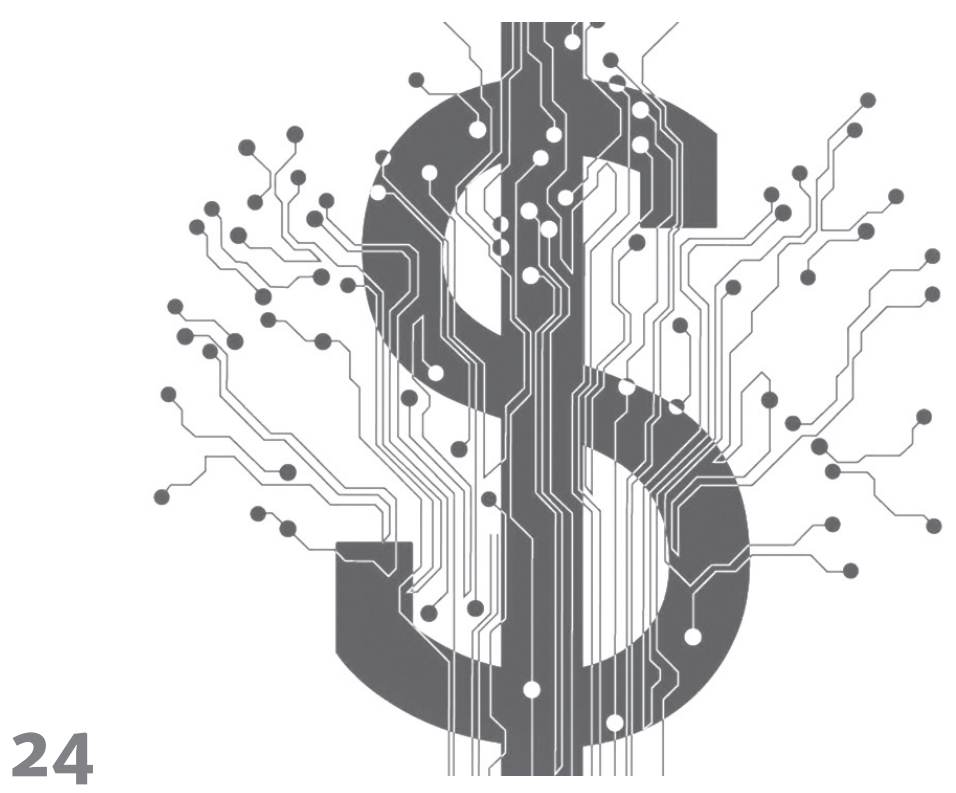

4. Producción baja de las prioridades nacionales

La obtención de nuevos conocimientos en CyT, se traduce un invento o innovación que mejore los procesos o productos de la econo- mía. La acumulación de estos recursos humanos se traduce en un incremento del coeficiente de inventiva y solicitudes de patentes por residentes nacionales. Sin embargo, este avance tiene dos límites: 1) falta de una cultura generalizada hacia la innovación y el registro de patentes comerciales y 2) carencia en el alineamiento estratégico de la producción científica y tecnológica.

Como se aprecia en el mapa 6, hay un aumento en la producción científica y tecnológica, sin embargo está concentrada en sectores específicos de la población y en regiones con alto potencial de desarrollo tecnológico, como la Ciudad de México y los principales centros urbanos.

Por otro lado, la falta de alineamiento estratégico en la producción científica, medida como la suma en la producción científica relativa a objetivos estratégicos sobre el avance general del conocimiento, muestra un estanca-

Mapa 6 Aportaciones estatales al Coeficiente de Inventiva nacional

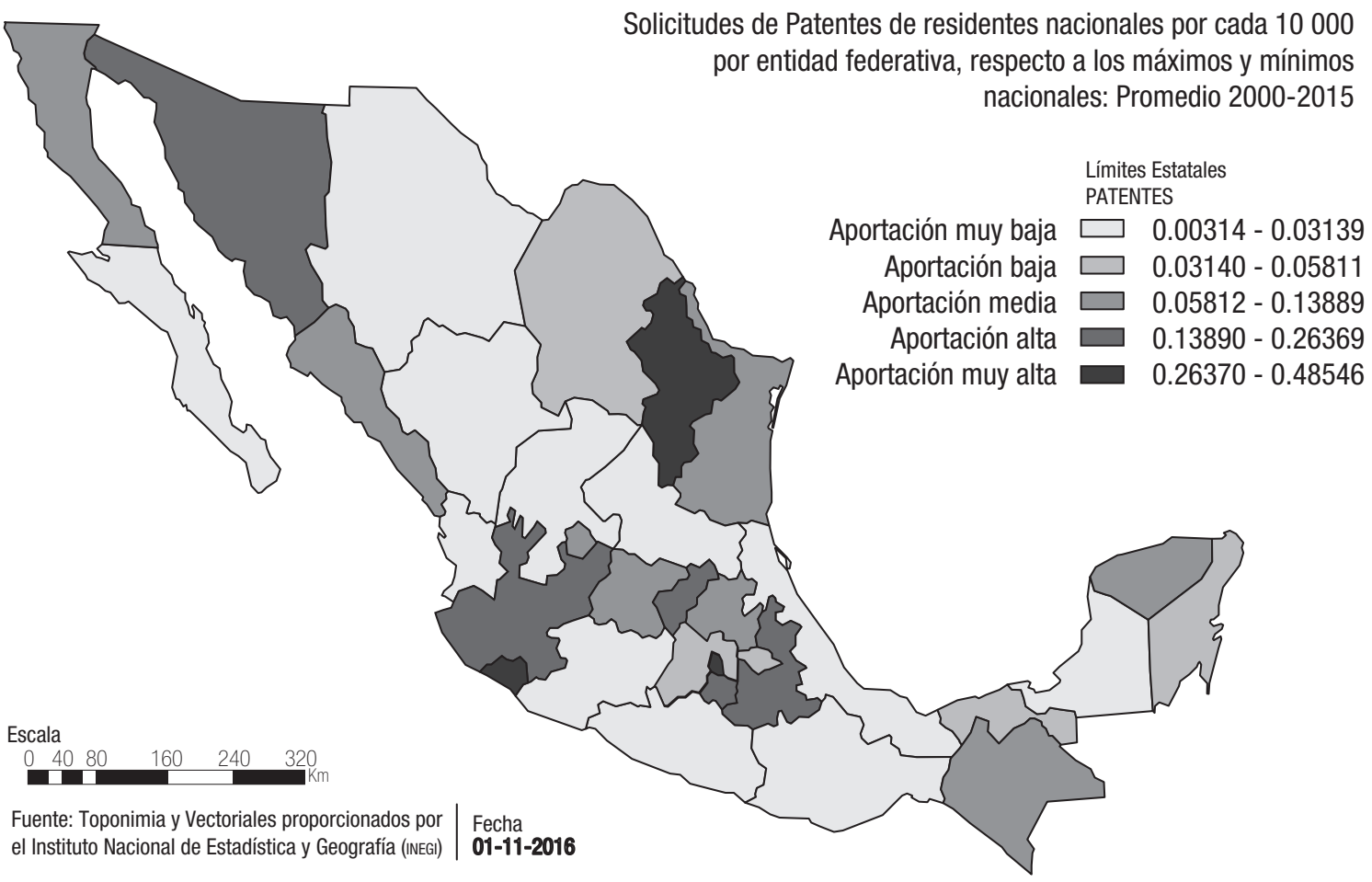


Rafael Buendía García | Jorge Pablo Rivas díaz | Ireiri Alonso León|

Evaluación del potencial del desarrollo en ciencia

y tecnología en México 2000-2015

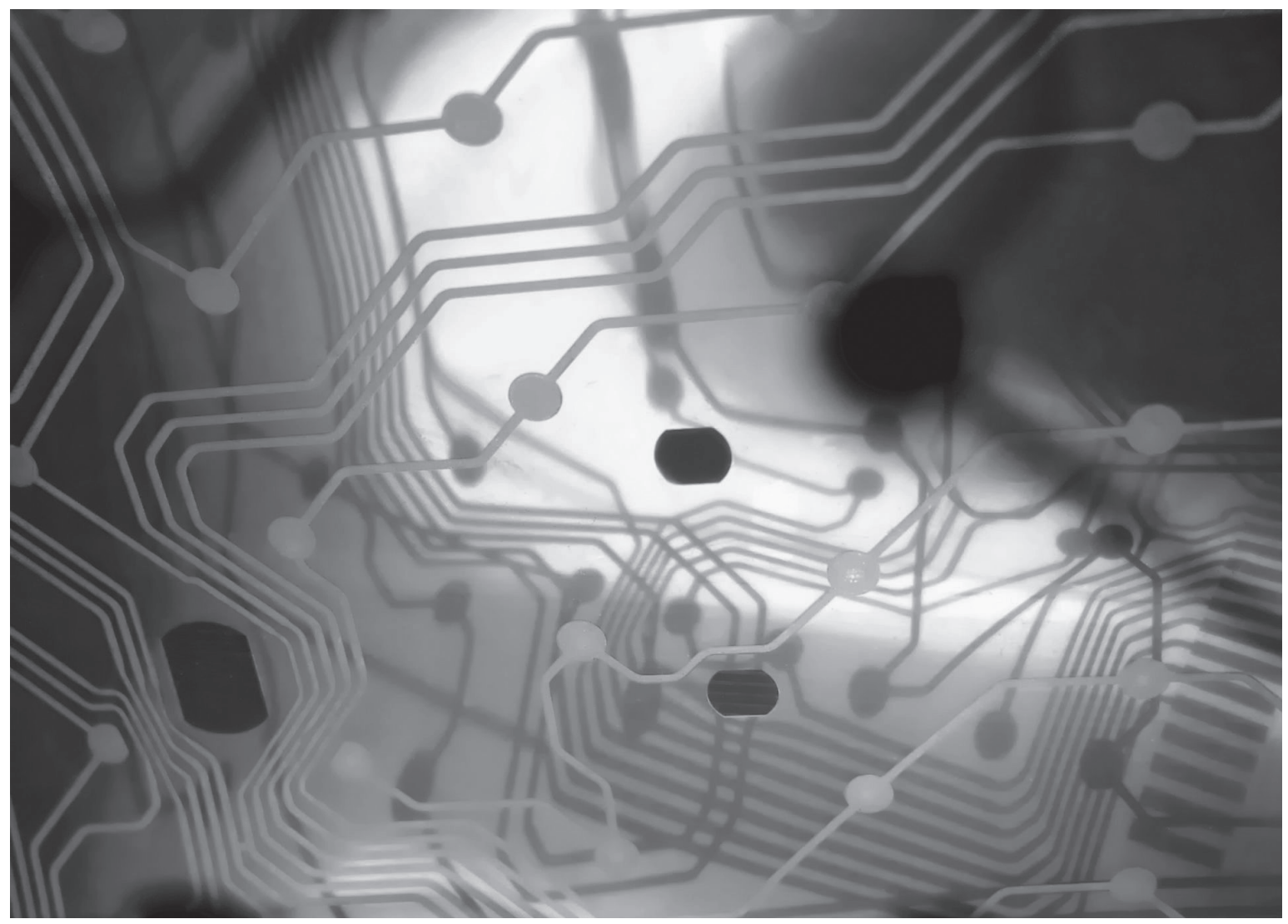

miento de $37 \%$ entre 2000 y 2015 , lo que frena la productividad y la eficiencia económica de la inversión en CyT, para los fines del desarrollo económico y social del país.

\section{Baja inversión y gasto público ineficiente}

Teniendo en cuenta que el gobierno promueve la producción científica y tecnológica, para México la participación financiera del sector público se caracteriza por tres limitaciones.

Primero, un bajo nivel de inversión del sector público en ciencia y tecnología, medida en relación del gasto federal en CyT como porcentaje del PIB, el cual alcanza en promedio durante el periodo de estudio un $0.37 \%$.

Segundo, una baja prioridad de la ciencia y la tecnología en el Gasto Programable del Sector Público Federal, que alcanza un promedio de $2 \%$ anual, es decir, se invierte de manera planeada y presupuestada en potencial de desarrollo económico futuro que lo que se gasta en desarrollo social o en gasto corriente de la administración del sector público.

Tercero, una alta concentración administrativa en la asignación del gasto público alrededor de las tareas del fomento a la producción científica y la formación de sus recursos humanos, que en el desarrollo de nuevas tecnologías enfocadas en los sectores estratégicos del país.

\section{Baja capitalización y dependencia tecnológica}

La integración de México al mercado globalizado genera otra limitación al progreso económico nacional. Dada la dependencia tecnológica de México al exterior y la baja producción interna de bienes de alta tecnología. El incre- 
mento en las relaciones comerciales con el exterior se traduce en un déficit en el saldo de la balanza de pagos tecnológica, la cual es deficitaria durante 2000 y 2015.

Por otra parte, el grado de capitalización del encadenamiento de la producción de recursos humanos, producción científica y tecnológica, y la innovación en procesos, productos y servicios, está sujeta a un bajo nivel de formalización de marcas comerciales, el cual representa una magnitud del 30\%, la cual es baja respecto al observado en Estados Unidos.

\section{Conclusiones}

A partir del análisis anterior se desprenden dos conclusiones sobre el estado de arte de la Ciencia y la Tecnología: 1) una alta concentración en los progresos agregados y 2) avances diferenciados entre los estados de la República, reforzándose la desigualdad en el potencial de desarrollo.

Además, del Índice se desprenden seis límites estructurales al progreso económico: 1) desequilibrios en la distribución espacial del potencial de desarrollo; 2) desarticulación de las etapas en la formación educativa; 3) recursos humanos especializados escasos y desequilibrados; 4) producción baja y desarticulada de las prioridades nacionales; 5) baja inversión y gasto público ineficiente y, 6) baja capitalización y dependencia tecnológica. Dichos límites y su relación entre sí, hacen que la eficiencia y el impacto del progreso en ciencia y tecnología sea poco significativo dentro del territorio nacional, puesto que existe una alta concentración y desigualdad en el potencial de desarrollo que merma los avances en materia de CyT.

Es por ello que el progreso en CyT sólo puede lograrse si se disminuyen los rezagos educativos en los distintos niveles educativos y se mejora su calidad, por lo que la política educativa nacional deberá considerar a la CyT como parte de sus programas educativos, encaminados a las necesidades y condiciones regionales, al igual que al desarrollo de recursos humanos especializados en las áreas estratégicas para el desarrollo nacional.

\section{Bibliografía}

López, S., La vinculación del sector de ciencia y tecnología con el sector productivo: su perfil económico, Ciudad de México: unam, 1996.

Aldana, M., ¿Qué le falta a la ciencia en México? Obtenido de UnAm, Instituto de Ciencias Físicas, 2012.

Disponible en: http://www.fis.unam.mx/ max/ MyWebPage/aldana_ciencia_en_mexico_temas_2012.pdf

Amieva, J., Finanzas Públicas en México, México, Porrúa, 2010.

Banco Mundial, La Enseñanza Superior: Las lecciones derivadas de la experiencia. Washington, D. C, The World Bank, 1995.

Becker, G., Human Capital: A Theoretical and Empirical Analysis with Special Reference to Education. USA, The University of Chicago Press, 1993.

Bekerman, M., Encadenamientos Productivos: Estilización E Impactos Sobre El Desarrollo De Los Paises Periféricos, Obtenido de Asociación Argentina de Econmía Política, 200. Disponible en: http:// www.aaep.org.ar/anales/works/works2001/bekerman_cataife.pdf

Bekкers, R., \& Isabel, B. F., Analyzing knowledge transfer channels between universities and industry: To what degree do sectors also matter?, Research Policy, 2010, 37(10), 1837-1853.

Didou, S., \& Gérard, E., Fuga de cerebros, movilidad académica, redes cientificas: Perspectivas latinoamericanas, 2009, obtenido de UNesCO: www.iesalc. unesco.org.ve/dmdocuments/biblioteca/libros/ fuga.pdf

Feldman, M. P., Link, A. N., \& Siegel, a. D., The Economics of Science and Technology: An Overview of 


\section{Rafael Buendía García | Jorge Pablo Rivas Díaz | Ireiri Alonso León|}

Evaluación del potencial del desarrollo en ciencia y tecnología en México 2000-2015

Initiatives to Foster Innovation, Entrepreneurship, and Economic Growth. Boston: Kluwer Academic Publishers, 2002.

Galindo, M., \& Ríos, V., Productividad. Obtenido de Serie de Estudios Económicos, Vol. 1, 2015, Disponible en: http://scholar.harvard.edu/ files/vrios/files/201508_mexicoproductivity. pdf?m=1453513201

Gibbons M. Limoges, C., Nowotry, H., Schwartzman, S., Scott, P., \& Trow, M., La Nueva producción del conocimiento. La dinámica de la ciencia de la investigación en las sociedades contemporáneas, Barcelona: Pomales-Corredor, 1997.

Justman, M., \& Teubal, M., A Structuralist Perspective on the Role of Technology in Economic Growth Development. 1991, obtenido de World Development: http://s3.amazonaws.com/academia.edu. documents/40318378/A_structuralist_perspective_on_the_role_20151123-8487-1a80lh6.pdf?A WSAccessKeyId=AKIAJ56TQJRTWSMTNPEA $\&$ Expires $=1479187265 \&$ Signature $=c F x B 6 n l R r A c$ pI5efvyOj7M5ALJE\%3D\&response-content-disp osition $=$ inline $\% 3$

Kıм, Y., Loayza, N., \& Meza-Cuadra, C. M., Productivity as the key to economic growth and development, 2016, obtenido de World Bank: http://documents. worldbank.org/curated/en/314741472533203058/ Productivity-as-the-key-to-economic-growthand-development

Mandujano, N., Federalismo fiscal en México: Una propuesta para fortalecer la hacienda pública estatal. México: unam, Instituto de Investigaciones Económicas, 2010.

Marx, K., El capital (3a ed., Vol. 1). México: Fondo de Cultura Económica.

Medina, S. (2004). La dependencia tecnológica en México, 1999, obtenido de Economía Informa: http://www.economia.unam.mx/publicaciones/ reseconinforma/pdfs/330/07SalvadorMedina.pdf

Molero, J., \& Buesa, M., Nationalist economic poli$c y$ and the importation of technology: a note on the Spanish case, 1940-1963, en I. y. Mándi, Emerging
Development patterns: European contributions, Budapest: European Association of Development Research and Trainng Institutes, Institute for World Economy, 1983.

ocDe, Oslo Manual, Propuesta de lineamientos para colección e interpretación de datos sobre investigación tecnológica. París, 1997.

OCDE, Innovation for Development, 2012, obtenido de OCDE: https://www.oecd.org/sti/inno/50586251. pdf

Osorio, J., Ciencia, tecnología e innovación en el desarrollo de México y América Latina, México, unAm e Instituto de investigaciones económicas, 2013.

Pacheco, A., La educación como promotora del desarrollo: el caso de la educación superior en México 19902012, México, UnAm, 2014.

Reamer, A., The Impacts of Technological Invention on Economic Growth - A Review of the Literature, 2014, obtenido de The George Washington Institute of Public Policy: https://gwipp.gwu.edu/files/ downloads/Reamer_The_Impacts_of_Invention on_Economic_Growth_02-28-14.pdf

Ricardo, D., Principios de economía política y tributación. (P. d. Braun, Trad.) Madrid: Pirámide, 2003.

Rosenberg, N., Innovation and economic growth, 2004, obtenido de OCDE: https://www.oecd.org/ cfe/tourism/34267902.pdf

Schumpeter, J. A., Teoría del desenvolvimiento económico. Una investigación sobre ganancias, capital, crédito, interés y ciclo económico, $4^{\mathrm{a}}$ ed., México-Buenos Aires, Fondo de Cultura Económica, 1967.

Silverberg, G., The Economics of Growth and Technical Change: technologies, nations, agents. England: Edward Elgar Publishing Company, 1994

Sмith, A. 1.-1., Investigación de la naturaleza y causas de la riqueza de las naciones. Barcelona, Planeta DeAgostini, 1997.

unesco, Documento de Politica para el Cambio y el Desarrollo de la Educación Superior, París, UNEsco, 1995. 
unesco, Heterogeneidad educativa, 2003, obtenido de UNESCO: http://www.buenosaires.iipe.unesco.org/ sites/default/files/informe18_heterogeneidad.pdf

Valdés, H., Segundo Estudio Regional Comparativo y Explicativo, 2006, obtenido de UNEsco: http://unesdoc.unesco.org/ images/0019/001902/190297s.pdf

Velho, L., La ciencia y los paradigmas de la política cientifica, tecnológica y de innovación, en Estudio social de la ciencia y la tecnología desde América Latina, México, Siglo del Hombre Editores, 2011.

Villaseca, E., Tecnología e innovación: Factores de Crecimiento Económico, 2004, obtenido de Asociación Española de Ciencia Regional : http://www. aecr.org/web/congresosAACR/2004/pdf/mesaC/ C1.pdf
WORLD BANK, Research for Development: A World Bank Perspective on Future Directions for Research, 2010, obtenido de World Bank: http:// www.felixpena.com.ar/contenido/negociaciones/ anexos/2010-10-research-for-development.pdf

\section{Referencias de Información y Documentos Oficiales}

Banco Mundial (2000 - 2014) Indicadores de Ciencia y Tecnología.

conacyt. Anexo Estadístico. Informe General del Estado de la Ciencia y la Tecnología (2000 - 2014); México.

SEP. Sistema Nacional de Información Estadistica Educativa. Indicadores y Pronósticos Educativos. Serie histórica de indicadores. México.

INEgI, Sistema de Cuentas Nacionales de México. 